\title{
Programmer Specified Pointer Independence
}

\author{
David Koes \\ CS Department \\ Carnegie Mellon University \\ Pittsburgh, PA 15213 \\ dkoes@cs.cmu.edu
}

\author{
Mihai Budiu \\ CS Department \\ Carnegie Mellon University \\ Pittsburgh, PA 15213 \\ mihaib@cs.cmu.edu
}

\author{
Girish Venkataramani \\ ECE Department \\ Carnegie Mellon University \\ Pittsburgh, PA 15213 \\ girish@cs.cmu.edu
}

\begin{abstract}
Good alias analysis is essential in order to achieve high performance on modern processors, yet precise interprocedural analysis does not scale well. We present a source code annotation, \#pragma independent, which provides precise pointer aliasing information to the compiler, and describe a tool which highlights the most important and most likely correct locations at which a programmer should insert these annotations. Using this tool we perform a limit study on the effectiveness of pointer independence in improving program performance through improved compilation.
\end{abstract}

\section{Categories and Subject Descriptors}

D.3.3 [Programming Languages]: Language Constructs and Features; D.3.4 [Programming Languages]: Processors-Compilers; D.3.4 [Programming Languages]: Processors-Optimization

\section{General Terms}

Performance

\section{Keywords}

Memory Performance, Alias Analysis, Pointer Independence

\section{INTRODUCTION}

Alias analysis, the identification of pointers which point to the same memory space, is an important part of any optimizing compiler. While numerous static alias analysis techniques exist (see [12] for a review), any static, intra-procedural analysis will be limited by its lack of knowledge of whole program behavior. However, it is possible for the programmer to provide this whole program knowledge by annotating the program suitably. An example of such an annotation is the restrict type qualifier that was introduced in the ANSI C99 standard [2]. In this paper, we investigate an alternative annotation that more closely relates pointer aliasing information to its use by optimization passes within a compiler. Section 3 describes the semantics of and motivation for our new \#pragma independent annotation. The implementation

Permission to make digital or hard copies of all or part of this work for personal or classroom use is granted without fee provided that copies are not made or distributed for profi t or commercial advantage and that copies bear this notice and the full citation on the fir rst page. To copy otherwise, to republish, to post on servers or to redistribute to lists, requires prior specifi $\mathrm{c}$ permission and/or a fee.

MSP'04, June 8, 2004, Washington, DC, USA.

Copyright 2004 ACM 1-58113-941-1/04/06 ...\$5.00. details for including the pragma in the compiler are described in Section 4.

In Section 5 we present an automated system for assisting programmers in appropriately annotating source code. The system uses static information (derived from code structure) and dynamic information (derived from profiling) to suggest to the programmer independent pointer pairs with a high payoff for improving program performance.

To estimate the efficacy of the pragma and our tool, we present performance numbers in Section 6. We evaluate the impact of the pragmas using gcc for both a simulated in-order single issue processor and the EPIC Intel Itanium processor. We perform a second evaluation using the CASH experimental compiler [3], targeting a reconfigurable architecture. In addition, we evaluate the ability of the tool to identify important pragmas for performance improvement and the amount of effort involved in validating the automatically discovered pragmas.

In Section 7 we conclude that programmer specified pointer independence is a reasonable and effective scheme for improving program performance, but both the compiler and the targeted hardware must have the means of taking advantage of the improved aliasing information.

\section{RELATED WORK}

Pointer analysis is an important part in any optimizing or parallelizing compiler as potentially aliasing memory references can introduce false dependencies which inhibit optimizations and reduce parrallelism. While much work has been done to improve the precision and efficiency of pointer analysis [12], an intra-procedural static pointer analysis can not take advantage of whole-program dynamic information. Inter-procedural pointer analysis performs a whole program analysis, but does not scale with program size without losing precision $[13,26]$ and is complicated by separate compilation and the use of library functions. Using our methodology, the programmer provides pointer independence information which the compiler uses directly, just as it would use the results of a complex and expensive alias analysis. The overhead in the compiler of supporting our method is therefore negligible.

Previous systems have used programmer annotations to provide memory aliasing information to the compiler or to analysis tools. In these systems the annotation is a type qualifier and the purpose is to aid in program understanding [1], program checking and verification [7, 8], or supporting type-safety [11]. In contrast, our annotation is not a type, but a precise statement of pointer independence. The compiler has no obligation to ensure the correctness of the annotations and the purpose of the annotations is simply to increase optimization opportunities and application performance. The ANSI C99 restrict type qualifier was designed to promote 
optimization [2], but does not directly map to information immediately usable by an optimizing compiler. Because restrict describes a more general aliasing property than our annotation, it is more difficult for both the programmer and compiler to reason about. The SGI MipsPro compiler provided an ivdep pragma which is used to break loop-carried dependencies between memory references in an inner loop. We describe a much more general approach. ASAP [15] is a language for describing aliasing properties within data structures. ASAP also relies upon the programmer to ensure correctness.

Another solution to the problem of overly conservative alias information is performing dynamic disambiguation at run-time. This can either be done completely in the compiler by generating instructions to check addresses [20] or by a combination of compiler and hardware support $[21,18]$. Hardware support allows the compiler to speculatively execute instructions under the assumption that memory references do not alias. If the assumption proves false, potentially expensive fix-up code is executed. A hardware based solution has the added advantage over software only approaches since it can successfully optimize cases where pointers alias, only infrequently. On the other hand, our proposal requires no special hardware and the final executable contains no extra instructions to check for aliasing.

The use of optional annotations in source code seems to be a very good trade-off between complex static analyses, expensive run-time checks, and the introduction of full-fledged new language mechanisms. For example, Linus Torvalds has advocated their large-scale use in the Linux kernel, mostly for security reasons [?].

Previous work has evaluated the effect of improved alias analysis on program performance $[9,14,6,10]$. The aggressive register promotion and memory redundancy elimination of CASH parallel these studies.

A significant contribution of this work is the automated toolflow for independent pointer discovery. This tool-flow uses both program structure information and run-time execution statistics to propose "interesting" pointer pairs to be further investigated by the programmer. We show that the tool is very focused and requires little programmer intervention to obtain most of the benefits of the precise aliasing information.

\section{3. \#PRAGMA INDEPENDENT}

We propose a pragma which allows the programmer to provide the compiler with precise and useful pointer independence information. The pragma has the syntax:

\#pragma independent ptr1 ptr2

This pragma can be inserted anywhere in the program where $p t r 1$ and $p t r 2$ are both in scope. The pragma directs the compiler to treat, within the scopes of ptrl and ptr2, any memory object accessed using ptrl as distinct from any memory object accessed using ptr2.

We also allow the use of the pragma with $n$ arguments, where $n>2$; this implies pairwise independence between all pointer pairs from the argument list. Since the multiple-argument form does not provide increased expressive power (it merely reduces the number of annotations required), it will not be discussed further.

As an example, consider the $\mathrm{C}$ code in Figure 1. We assume that pairwise independence exists between the pairs $(a, b)$ and $(a, c)$ but nothing can be said about the relationship between $b$ and $c$. The Itanium assembly code generated from this example by a pragmaaware $\mathrm{gcc}$ is shown in Figure 2. Using the programmer provided pointer independence information, the compiler successfully removes an unnecessary store to a.

The independence pragma is easy to use and reason about, since the programmer only has to take into account the behavior of two

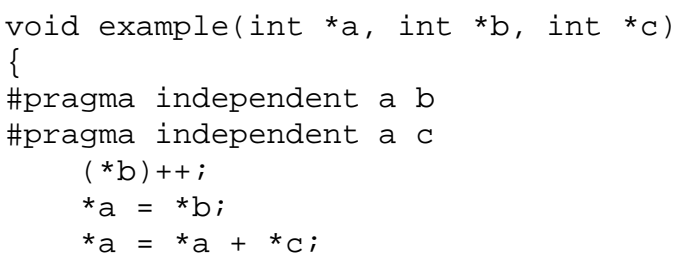

Figure 1: Pragma usage example. The programmer has informed the compiler that the pairs $(a, b)$ and $(a, c)$ will never alias, but makes no claims about the relationship between $b$ and $c$.

pointers. Furthermore, this type of information is exactly what an optimizing compiler needs when performing code motion optimizations such as partial redundancy elimination (PRE) and instruction scheduling.

\section{USING \#PRAGMA INDEPENDENT IN THE COMPILER}

We have added support for the independence pragma to both gcc (version 3.3) and to the Compiler for Application Specific Hardware (CASH) [3]. Within gcc, we have modified the frontend to parse \#pragma independent and, for each pointer variable declaration, maintain a list of pointer variables which have been declared to be independent of that pointer. Within the alias analysis initialization phase of the gcc back-end, we then propagate this information to compiler temporaries. Since independent pointers must point to completely independent memory objects, we also propagate the independence information through address calculations. For example, $p$ and $p+3$ are assumed to point within the same "object", and thus the independence information valid for $p$ is assumed to be valid for $p+3$ as well. Also, if $p$ is assigned to $q$, we propagate whatever independence information we have from $p$ to $q$ as well. Finally, when gcc's optimization passes query for pairwise pointer independence, we use the independence information if possible. Pointer independence information is used by $\mathrm{gcC}$ in the CSE/PRE and instruction scheduling passes. Unfortunately, gCc does not have a register promotion optimization pass, which has been shown to benefit significantly from improved pointer independence information $[19,22]$. Overall, relatively little code is needed to add full support for the independence pragma to a conventional compiler (less than 200 lines of code).

We also added support to the SUIF [25] front-end which is used by CASH. The SUIF [25] front-end uses \#pragma independent statements to annotate the corresponding variable symbols. A dataflow analysis pass propagates the independence information through compiler temporaries and pointer expressions. The CASH back-end contains a memory disambiguator which attempts to prove that memory operations do not alias; the disambiguator was modified to query independence pragma information. The compiler can then take advantage of the increased parallelism in the dependency graph. Because the reconfigurable target can execute an arbitrary number of operations at once, increasing the parallelism in the compile-time dependency graph directly results in an increase in parrallelism at run-time.

\section{AUTOMATED ANNOTATION}

We have developed two systems for partially automating the annotation of source code with independence pragmas. Both systems combine a compiler's static analysis with runtime information to provide a ranked list of pairs of pointers that are candidates for 
Without pragma

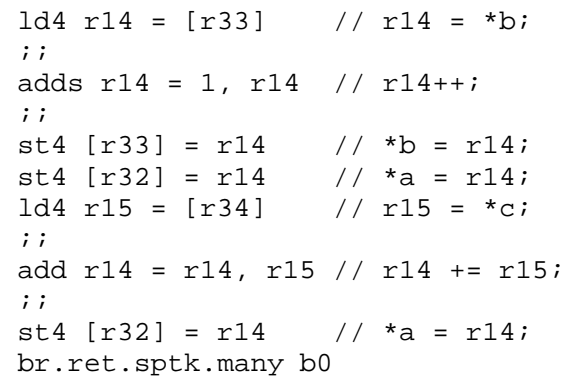

With pragma

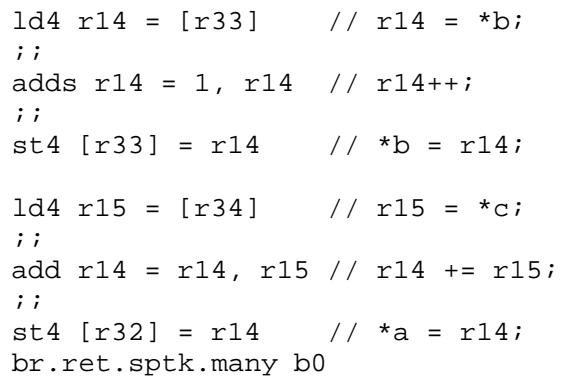

Figure 2: Itanium assembly code generated from the source in Figure 1. The double semicolons separate concurrent instruction groups. Using the information from the independence pragma, the compiler can remove a store instruction. On the Itanium processor, this avoids a split issue in the third instruction group, reducing the cycle time of the function.

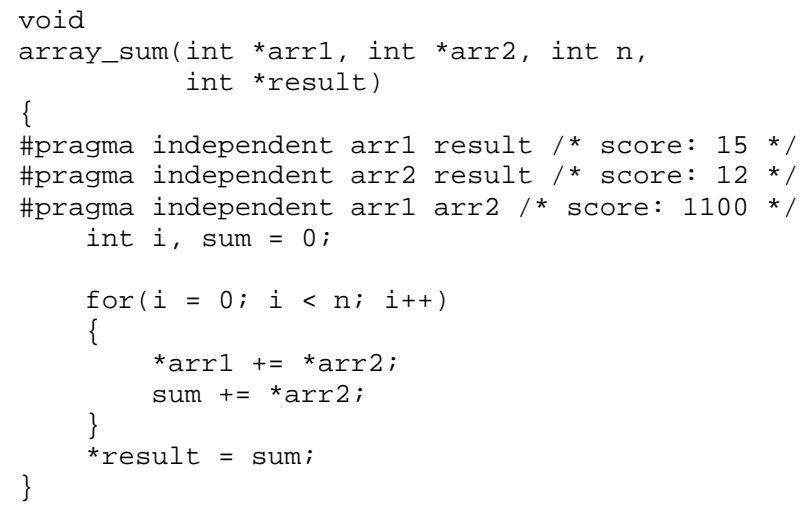

Figure 3: Sample code with pragma annotations and scores as produced by our tool-flow.

being marked as independent. Although the systems make an effort to eliminate aliasing pointer pairs from consideration, it is ultimately the programmer's responsibility to evaluate these candidates for correctness.

Figure 3 shows a code snippet which has been automatically annotated with candidate independence pointer pairs. The scores estimate the effect of making the pair independent on program performance. These scores, as described below, summarize both information about the static code structure and execution frequencies. The pair (arr1, $\operatorname{arr} 2)$ has a much higher score than the other two pairs since these pointers are both accessed within the loop body. Knowing that they are independent allows the compiler to load the values of $* \operatorname{arr} 1$ and $\star \operatorname{arr} 2$ into registers for the whole loop execution (perform register promotion). The pair (arr1,result) has a higher score than the pair (arr2,result), reflecting the fact that there is an opportunity to schedule the stores to $\operatorname{arr} 1$ and result in parallel after register promotion.

The code fragment in Figure 3 was automatically annotated by using the tool-flow depicted in Figure 4. Of course, nothing prevents the function array_sum from being called with pointers that point to overlapping memory regions as the arguments $\arg 1$ and arg2. Although the tool-flow checks whether this ever occurs for the profiling input sets, this is no guarantee of code correctness. It is the responsibility of the programmer to verify the correctness of the annotations by inspecting all the call sites of array_sum. The annotation scores serve as a guide to the programmer, focusing attention on the pairs which are most likely to bring performance benefits. As we show in Section 6, the scores closely track the 90-
10 rule of program hot-spots (there are very few hot annotations) and verifying an annotation is not a time consuming task. Programmer effort for checking annotations is thus focused on the important pairs.

Within CASH, memory dependencies are first-class objects represented by token edges [4]. As shown in Figure 5, if two memory accesses may interfere, a token edge is inserted between them in the internal dataflow representation of CASH. A memory operation must wait for all incoming tokens before it can access memory. Once the memory operation has been performed a token is generated, permitting other memory operations to take place. In this way memory dependencies are turned into dataflow.

A memory disambiguation pass examines every token edge and, using traditional intra-procedural pointer analysis, eliminates edges between memory references that can be proven not to alias. The base pointers of memory references that the analysis determines might alias are marked as candidates for annotation. Every pair of pointers is associated with a static score which estimates, for that pair, the benefit of declaring that pair as independent. For example, a pair which prevents an important optimization from taking place would receive a high score. There are many possible ways to compute a relevant score using only static information. Our current implementation uses a simple heuristic: the score of a pointer pair $(a, b)$ is the number of token edges between memory accesses to a and to $\mathrm{b}$.

gcc statically scores each pointer pair with the number of times gCC's optimization passes query for independence information between the two pointers.

Although in many cases the two compilers find the same candidate pointer pairs, in some cases each compiler will find a pair the other does not. The CASH compiler is better at finding pointer pairs that are inhibiting optimizations, especially when the pointers are separated by complicated control flow. The gcc compiler will sometimes find pointers which CASH, with its more sophisticated alias analysis, can identify as not aliasing or not impacting optimization (if both pointers are used only in loads, for example).

Since pairs are aggressively generated without using inter-procedural analysis, some pairs will alias at run-time and therefore should not be annotated as independent. Thus, both CASH and gCC also instrument the program to collect run-time information: for each pointer pair, a special check operation is inserted. This check operation acts as both an aliasing check and a frequency counter. When the program is run, the check records any pointer pairs that alias, and thus are not independent. The frequency counter is used to identify frequently-executed code. With CASH, the check operation is an instruction implemented directly within the simu- 


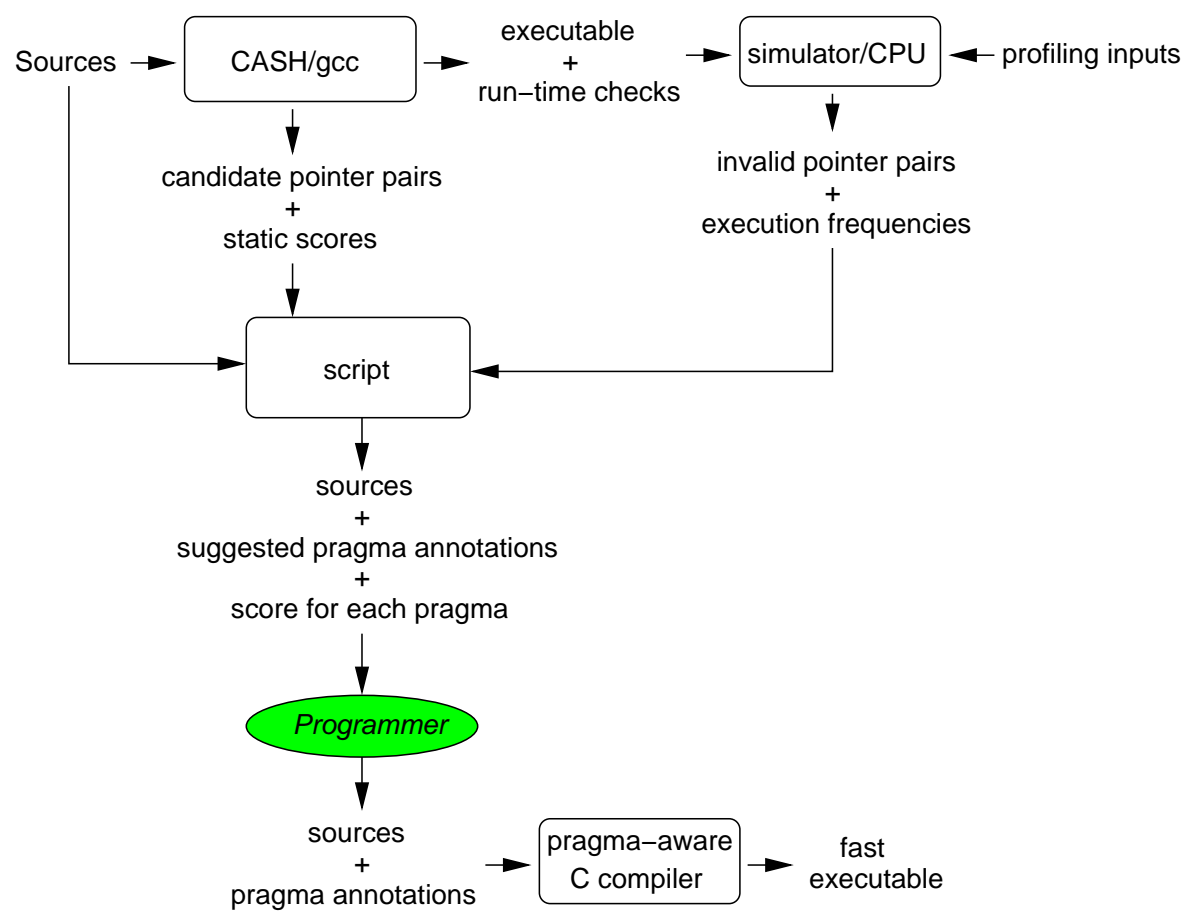

Figure 4: Tool-flow for independence pragma source annotation. Notice that the programmer is part of the tool-flow, certifying the correctness of the suggested annotations.

lator whereas with $\mathrm{gcc}$ it is a function call to a library function performing a dynamic check.

The compile-time and run-time information are combined by the tool. This weeds out the pairs which were discovered to alias and computes an overall score using both the static score and the frequency counts for each pair (currently by multiplying them). The script sorts the annotations by the overall score, and can optionally annotate the original source code with the annotations whose scores are above a certain programmer-selected threshold; this is how the code in Figure 3 was produced. The programmer can then focus only on analyzing the source code having pairs with high overall scores.

\section{RESULTS}

\subsection{Evaluation}

We have evaluated the effectiveness of our automated annotation system and the ability of the modified compilers to take advantage of the independence information on three very different machine models: (1) We used our modified version of gcc to compile to the MIPS-like SimpleScalar [5] architecture which we then simulated running on an in-order, single issue processor. (2) We used the same gcc version to compile for a 733Mhz EPIC Intel Itanium processor [16]. Programs were compiled using the optimization flags -O2 -funroll-loops. (3) Finally, we used our CASH compiler to target a reconfigurable fabric. Our results are obtained from the programs in Mediabench [17], Spec95 [23], and Spec2000 [24]. When possible we ran the annotation tool on the training sets and collected performance results from the reference sets. Both the CASH and gcc generated annotations were used for the reconfigurable target while only the gcc generated ones were used for the SimpleScalar and Itanium targets.
Our two simulators provide cycle-accurate measurements, but are about three orders of magnitude slower than native execution. The measurements on the real Itanium system are plagued by variability from low-resolution timers and system activity. We have thus used different input sets for the simulated and real system (short ones on simulators, large ones on the real system). In addition, we do not produce results for large benchmarks with the simulators nor results from smaller benchmarks on the Itanium.

The source code of all benchmarks has been annotated with independence pragmas using the automated system. Although we have inspected and verified some benchmarks, we have not manually inspected every individual pragma that the system produces. All benchmarks produce the correct output when run with the annotations.

\subsection{Speed-ups}

The execution speed-up for annotated code on the in-order, single issue simulated processor is mostly negligible with only one benchmark (124.m88ksim) receiving a meaningful speed-up (13\%). This architecture is incapable of taking advantage of additional memory parallelism. Furthermore, the gcc SimpleScalar PISA back-end is somewhat rudimentary. Few target specific optimizations are performed and the underlying machine model does not accurately or precisely describe the actual machine model.

The execution speed-up for annotated code on the Itanium is shown in Figure 6. As expected, the highly parallel Itanium processor does better than the in-order SimpleScalar processor. $124 . \mathrm{m} 88 \mathrm{ksim}$ shows a speed-up of $1.28,177$. mesa a speedup of 1.09, and 132. i jpeg a speed-up of 1.02. The remaining benchmarks either did not show a significant speed-up, or had too short a running time to be measured precisely. The observed improvements appear to be exclusively a result of improved instruction scheduling suggesting a more sophisticated Itanium compiler 

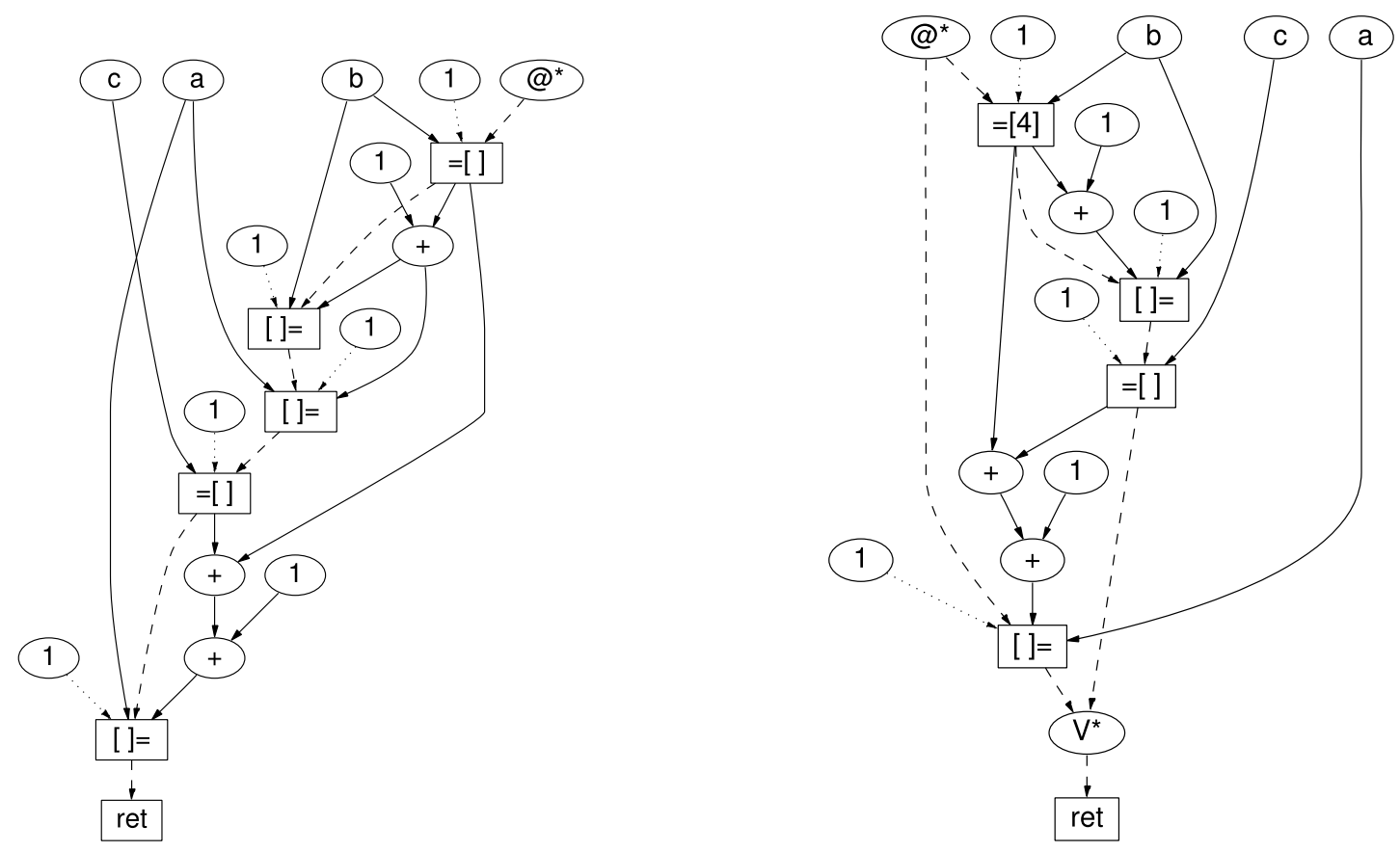

Figure 5: CASH internal representation of Figure 1. On the left is the code with no pragmas while on the right is the code with pragmas. Operations are represented rectangles and inputs by ellipses. An operation does not execute until all of its input are available, including token inputs. Load operations are shown as $=$ [ ] ; each has 3 inputs: address, predicate, and token and two outputs: data and token. Store operations are shown as [ ] =; each has 4 inputs: address, data, token, and predicate, and one token output. Dashed lines represent tokens (memory dependences); “@*” is the “input token”. The V node "joins" tokens. Dotted lines represent predicates. Note that the graph on the right has one fewer store.

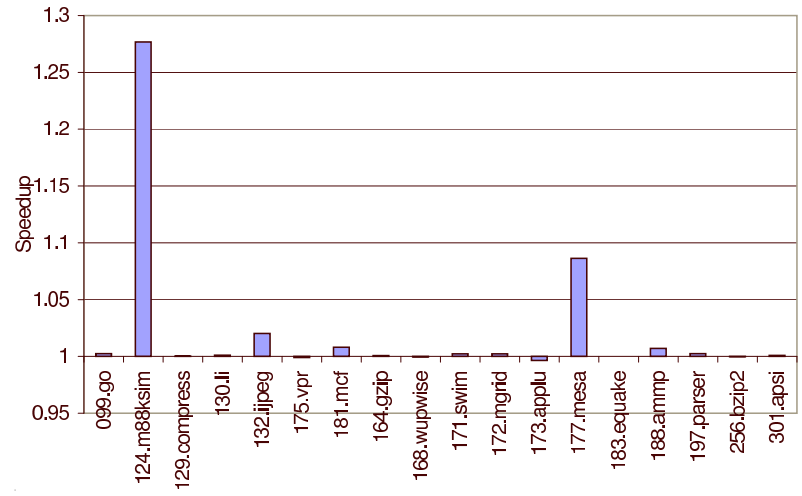

Figure 6: Speed-up using \#pragma independent annotated code compiled with $\mathrm{gcc}$ for an Intel Itanium processor.

would demonstrate more benefit (as in [10]). Furthermore, there are only a few critical pragmas in each benchmark. For example, in $124 . \mathrm{m} 88 \mathrm{ksim}$ there is just one pragma that accounts for all the observed speed-up; it breaks dependencies within a memcpy-like loop. In 177 . mesa, three pragmas inside a critical function with pointer arguments are enough to account for all of the speed-up.

The execution speed-up for annotated code compiled for a reconfigurable fabric is shown in Figure 7. Most benchmarks demonstrate meaningful speed-ups with the most significant being speedups of $2.00,1.93,1.74,1.35$ for adpcm_d, jpeg_d, adpcm_e, and gsm_d respectively.

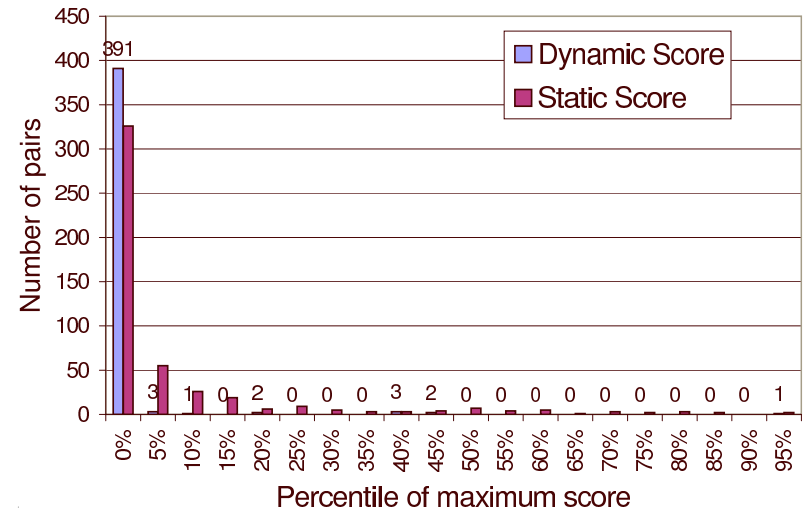

Figure 8: Score histogram for 132 . i jpeg.

\subsection{Scoring}

One goal of our tool is to give the programmer a way to pass information to the compiler without increasing the programming burden. To this end we evaluated the effectiveness of our tools in guiding the programmer effort toward the most profitable code regions.

Histograms of the scores of the pragmas, like the one in Figure 8 for 132. i jpeg, look surprisingly similar across all programs. Figure 8 shows histograms of both the static and dynamic scores. The $x$ axis is the normalized score of an annotation, binned in 20 equal intervals. The $y$ axis represents the number of annotations that have a score within 5\% of the $x$ value. For example, the 5\% bar labeled "dynamic", with a value of 3 , shows that 3 annotations 


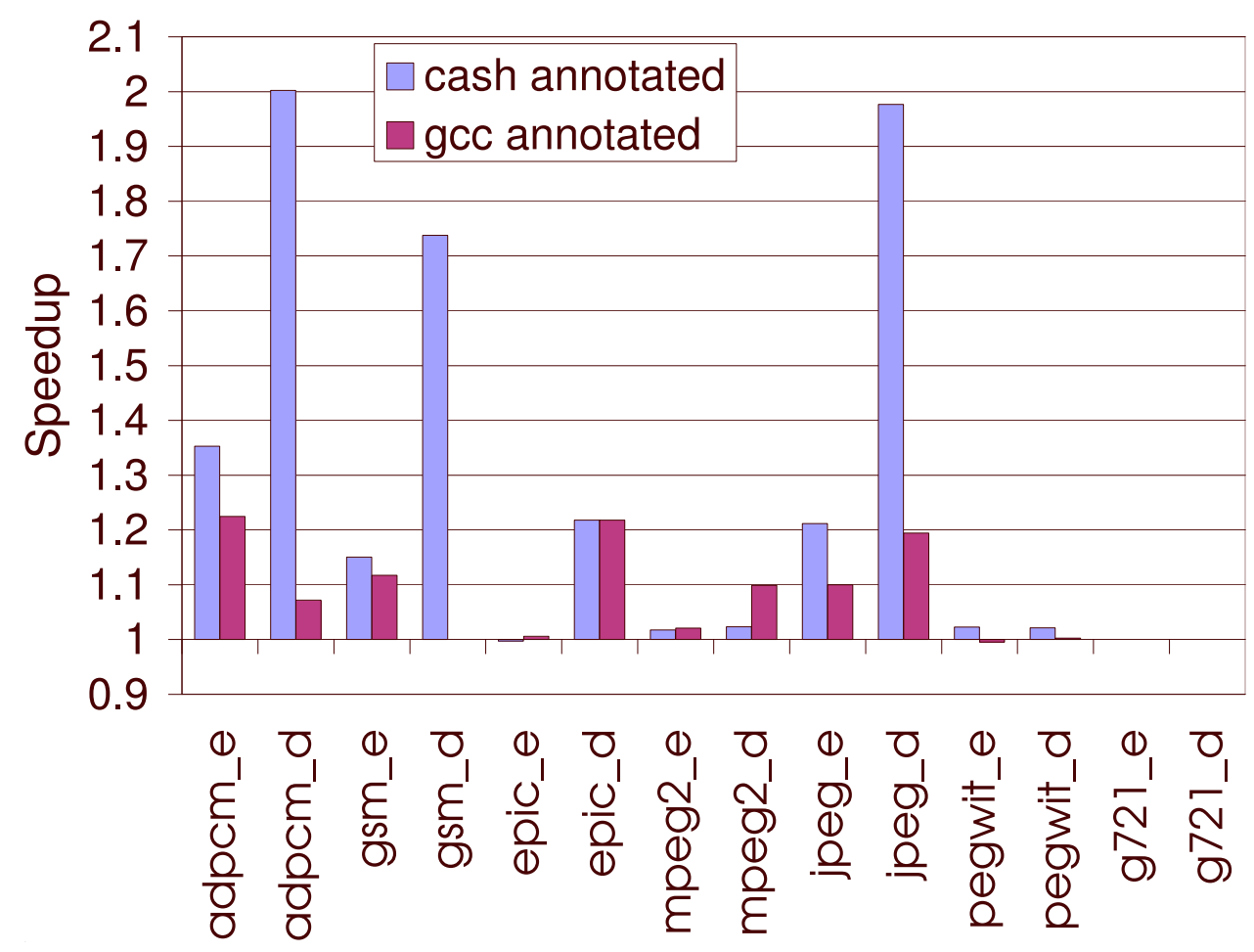

Figure 7: Speed-up using \#pragma independent annotated code compiled with the CASH compiler for a simulated reconfigurable fabric.

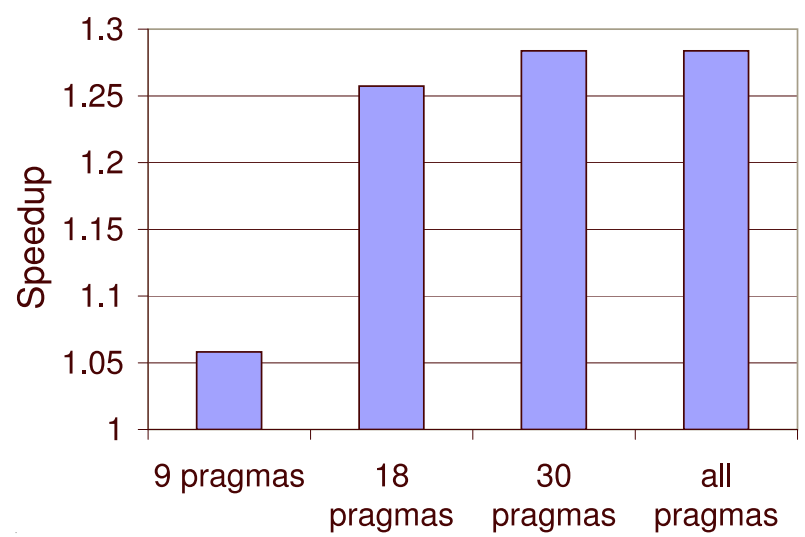

Figure 9: Speed-up for 132 . i jpeg run on a simulated reconfigurable fabric as a function of the number of pragmas used. Pragmas were added in order of decreasing importance.

have a score between $5 \%$ and $10 \%$ of the maximum score found. Both distributions have a sharp knee, which suggests a cut-off point for useful annotations. In this example, 22 pragmas account for the top $96 \%$ of the scores. These are the most likely to require the attention of the programmer. And in fact, as Figure 9 shows, the top 18 pragmas account for almost all of the improvement that can be gained.

In Table 1 we give the pragma counts found by our gcc-based automatic instrumentation system. The first three columns show the total number of pragmas inserted, the number of pointer pairs that were executed at least once for the given input set, and the number of pairs that were found to alias, thus whose annotations are incorrect. The fourth column shows how many of the correct annotations are below the "knee" of the curve (these were manually estimated by looking at the score distribution).

In order to verify that the high scoring annotations are indeed the most important we have carried out two experiments. We annotate each program with only a small number of annotations, the ones with the highest scores. Figure 10 presents results for all benchmarks for which we manually inspected only the highest ranking annotations (see below). Interestingly, in some cases using only a subset of the pragmas resulted in larger performance gains (mpeg2_d, pegwit_d). In these cases it appears that the added memory parallelism did not shorten the critical path, but did increase the cost of synchronization (as represented by the $\mathrm{V}$ node in Figure 5).

\subsection{Validation}

We tested our claim that manually validating an automatically generated pragma is not an onerous task (even if the programmer is unfamiliar with the code) by having several programmers verify some of the annotated source. The programmers were instructed to verify only the highest scoring annotations. The results are shown in Table 2.

Even though they had little experience with the code, it took an average of less than 2 minutes per pragma to validate its correctness. Most of the time was spent exploring the call tree to determine the origins of pointer arguments to functions. In addition, as the programmer became more acquainted with the structure and conventions of a benchmark, validation took less time. Some program constructs made validation difficult or impossible. In this case the annotation was marked as incorrect. We expect that a programmer with a deeper understanding of the code would be able to verify annotations almost instantaneously. 


\begin{tabular}{|c|c|c|c|c|c|c|c|c|c|}
\hline Bench & total & checked & conflict & useful & Bench & total & checked & conflict & useful \\
\hline 124.m88ksim & 119 & 57 & $\overline{2}$ & 12 & mesa & 979 & 107 & 9 & 25 \\
\hline 129.compress & 3 & 3 & 0 & 3 & mpeg2_d & 94 & 64 & 0 & 3 \\
\hline 130.li & 56 & 21 & 3 & 6 & mpeg2_e & 72 & 21 & 4 & 9 \\
\hline 132.ijpeg & 490 & 142 & 8 & 22 & pegwit_d & 34 & 24 & 3 & 11 \\
\hline 134.perl & 744 & 267 & 42 & 22 & pegwit_e & 34 & 25 & 4 & 14 \\
\hline 175.vpr & 188 & 39 & 4 & 12 & 176.gcc & 3470 & 2406 & 504 & 44 \\
\hline 181.mcf & 132 & 60 & 7 & 14 & 197.parser & 159 & 144 & 38 & 12 \\
\hline adpcm_d & 12 & 3 & 0 & 6 & 256.bzip2 & 40 & 36 & 34 & 3 \\
\hline adpcm_e & 12 & 3 & 0 & 6 & 300.twolf & 451 & 173 & 52 & 27 \\
\hline epic_d & 41 & 11 & 7 & 11 & 168.wupwise & 3 & 3 & 0 & 3 \\
\hline epic_e & 32 & 22 & 3 & 13 & 171.swim & 0 & 0 & 0 & 0 \\
\hline g721_d & 0 & 0 & 0 & 0 & 172.mgrid & 7 & 7 & 1 & 5 \\
\hline g721_e & 0 & 0 & 0 & 0 & 173.applu & 2 & 2 & 2 & 0 \\
\hline gsm_d & 36 & 10 & 1 & 9 & 177.mesa & 950 & 94 & 8 & 37 \\
\hline gsm_e & 36 & 21 & 4 & 11 & 183.equake & 30 & 13 & 2 & 6 \\
\hline jpeg_d & 418 & 90 & 2 & 12 & 188.ammp & 252 & 82 & 11 & 11 \\
\hline jpeg_e & 453 & 68 & 9 & 10 & 301.apsi & 463 & 362 & 3 & 14 \\
\hline
\end{tabular}

Table 1: Annotations statistics. The columns represent: benchmark name, total pointer pairs instrumented, pointer pairs with at least one run-time check, pointer pairs found to alias at run-time, number of most likely useful pointer pairs (knee of histogram curve). The last column is the number of pragmas that would most likely bring the highest benefit, and would thus be the best targets for manual validation.

\begin{tabular}{|l|rrr|rr|}
\hline Bench & lines & inspected & correct & time (min) & time/pragma \\
\hline adpcm_d & 307 & 8 & 8 & 5 & $<1$ \\
adpcm_e & 307 & 8 & 8 & 2 & $<1$ \\
gsm_e & 5841 & 9 & 9 & 25 & 2.8 \\
gsm_d & 5841 & 5 & 5 & 9 & 1.8 \\
epic_d & 2528 & 9 & 9 & 33 & 3.7 \\
epic_e & 2830 & 21 & 20 & 11 & $<1$ \\
mpeg_d & 10596 & 58 & 34 & 113 & 1.9 \\
mpeg_e & 7791 & 10 & 10 & 8 & $<1$ \\
jpeg_e & 27496 & 32 & 31 & 90 & 2.8 \\
jpeg_d & 27496 & 13 & 13 & 10 & $<1$ \\
pegwit_e & 6944 & 26 & 26 & 24 & $<1$ \\
pegwit_d & 6944 & 26 & 26 & 2 & $<1$ \\
mesa & 67081 & 30 & 27 & 16 & $<1$ \\
\hline
\end{tabular}

Table 2: Manual verification effort, expressed in minutes. For each benchmark we list the number of lines of source code, the number of automatically generated pragmas, the number of pragmas manually inspected (if there were many generated pragmas, only the highest ranking were inspected), the number of pragmas ascertained correct, and the times spent on validation: overall, and per-pragma. 


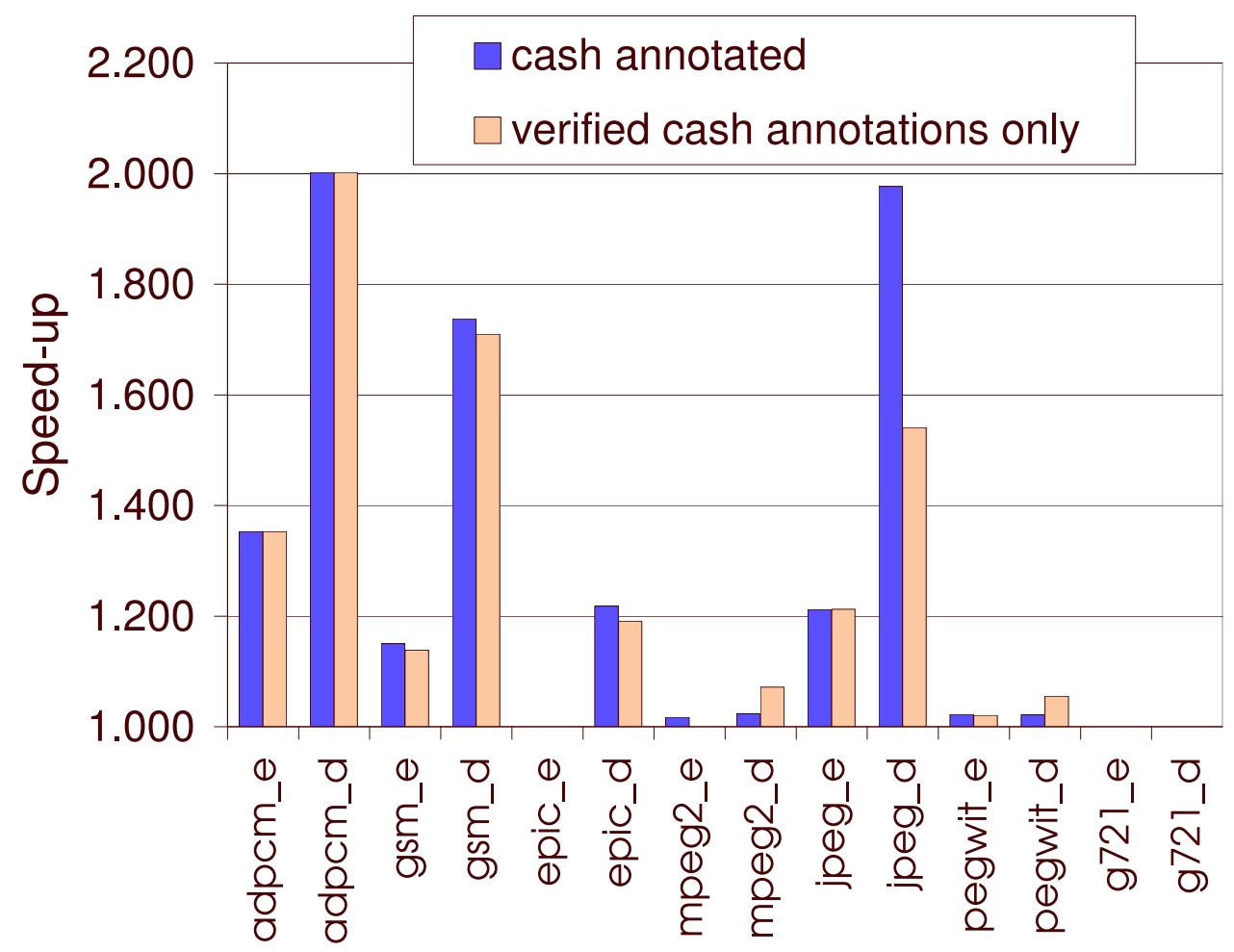

Figure 10: Speed-up due to pragmas on the reconfigurable hardware platform. First bar uses all pragmas (and is potentially unsafe); the second bar uses the highest-ranking pragmas that were manually certified to be safe.

\begin{tabular}{|l|rrr|}
\hline & local & argument & global \\
\hline local & 30 & 51 & 24 \\
argument & & 92 & 29 \\
global & & & 9 \\
\hline
\end{tabular}

Table 3: The types of pointer pairs verified. Of the 235 pairs that were manually inspected, we classified each verified pair by the defining scope of its members. A local pointer variable was always counted as a local reference, even if it could be proved to always equal a pointer argument.

Table 3 shows the counts for the different relationships between the verified pointers. Not surprisingly, few pointer pairs were between global references as the compiler can almost always differentiate the pointers in this case. Without inter-procedural analysis, function arguments, and local variables whose values come from function arguments, are the most likely candidates for programmer specified pointer analysis.

\section{CONCLUSION}

Uncertainty about pointer relationships and the inability to perform whole program analysis frequently handicaps compiler optimizations, particularly for languages like C. However, it is frequently the case that the programmer has knowledge about pointers that could help the optimizer, but the language provides no convenient mechanism for expressing this type of information. In this paper we have presented a mechanism that enables the programmer to specify to the compiler that certain pointers access disjoint memory regions and quantified the benefits that can be derived from exploiting this mechanism. We have also presented a tool-chain that uses compile-time and run-time information to suggest to the program- mer a small number of pointer pairs for which independence information could have a big impact on program performance. While these suggested pairs require manual verification, we have shown that even an investment of 10 minutes of code analysis can provide significant benefits.

The reliance upon manual verification is the single biggest drawback of our approach. If the code is improperly verified, or if correct code is later changed to be incorrect, subtle errors could be introduced. Given the flexibility and power of the C language, we feel that the additional burden to the programmer is relatively little, especially in light of the performance benefits. If automatic verification is desired, or if our approach were to be used in a language with a different paradigm than $\mathrm{C}$, an expensive inter-procedural analysis could be performed at link time. Since this analysis would only have to verify the annotated pointers, which consist of just those with the largest impact upon performance, and not perform any additional optimizations, such an approach would be considerable more efficient than a fully automatic approach that performed all pointer analysis and optimization at link time.

Allowing programmers to provide pointer independence information can result in meaningful increases in performance provided that the compiler and targeted architecture are capable of taking advantage of such information. Programmer specified pointer independence is a scalable, effective alternative to inter-procedural pointer analysis. Conventional compilers (such as gcc) lack sophisticated memory optimizations as such optimizations require expensive pointer analyses. Our approach removes this barrier to optimization at the cost of some programmer effort.

\section{ADDITIONAL AUTHORS}

Additional author: Seth Copen Goldstein (Carnegie Mellon University, email: seth@cs. cmu.edu). 


\section{REFERENCES}

[1] J. Aldrich, V. Kostadinov, and C. Chambers. Alias annotations for program understanding. In Object-Oriented Programming, Systems, Languages, and Applications (OOPSLA), November 2002., 2002.

[2] ANSI. Programming languages - C, 1999.

[3] Mihai Budiu and Seth Copen Goldstein. Compiling application-specific hardware. In Proceedings of the 12th International Conference on Field Programmable Logic and Applications, Montpellier (La Grande-Motte), France, September 2002.

[4] Mihai Budiu and Seth Copen Goldstein. Optimizing memory accesses for spatial computation. In Proceedings of the 1st International ACM/IEEE Symposium on Code Generation and Optimization (CGO 03), San Francisco, CA, March 23-26 2003.

[5] Doug Burger and Todd M. Austin. The SimpleScalar tool set, version 2.0. In Computer Architecture News, volume 25 (3), pages 13-25. ACM SIGARCH, June 1997.

[6] Ben-Chung Cheng and Wen-Mei W. Hwu. Modular interprocedural pointer analysis using access paths: design, implementation, and evaluation. In Proceedings of the ACM SIGPLAN 2000 conference on Programming language design and implementation, pages 57-69. ACM Press, 2000.

[7] David Evans. Static detection of dynamic memory errors. In SIGPLAN Conference on Programming Language Design and Implementation (PLDI '96), 1996.

[8] Jeffrey S. Foster, Tachio Terauchi, and Alex Aiken. Flow-sensitive type qualifiers. In Proceeding of the ACM SIGPLAN 2002 Conference on Programming language design and implementation, pages 1-12. ACM Press, 2002.

[9] Rakesh Ghiya and Laurie J. Hendren. Putting pointer analysis to work. In Proceedings of the 25th ACM SIGPLAN-SIGACT symposium on Principles of programming languages, pages 121-133. ACM Press, 1998.

[10] Rakesh Ghiya, Daniel Lavery, and David Sehr. On the importance of points-to analysis and other memory disambiguation methods for $\mathrm{C}$ programs. In Proceedings of the ACM SIGPLAN 2001 conference on Programming language design and implementation, pages 47-58. ACM Press, 2001.

[11] Dan Grossman, Greg Morrisett, Trevor Jim, Michael Hicks, Yanling Wang, and James Cheney. Region-based memory management in Cyclone. In Proceedings of the ACM Conference on Programming Language Design and Implementation, June 2002.

[12] Michael Hind. Pointer analysis: haven't we solved this problem yet? In Proceedings of the 2001 ACM SIGPLAN-SIGSOFT workshop on Program analysis for software tools and engineering, pages 54-61. ACM Press, 2001.

[13] Michael Hind, Michael Burke, Paul Carini, and Jong-Deok Choi. Interprocedural pointer alias analysis. ACM Transactions on Programming Languages and Systems, 21(4):848-894, 1999.

[14] Michael Hind and Anthony Pioli. Evaluating the effectiveness of pointer alias analyses. Sci. Comput. Program., 39(1):31-55, 2001.

[15] Joseph Hummel, Laurie J. Hendren, and Alexandru Nicolau. A language for conveying the aliasing properties of dynamic, pointer-based data structures. In Proceedings of the 8th International Parallel Processing Symposium, pages
208-216, Cancun, Mexico, April 1994. IEEE Computer Society.

[16] Intel Corporation. Intel Itanium 2 Processor Reference Manual, 2002.

[17] Chunho Lee, Miodrag Potkonjak, and William H. Mangione-Smith. MediaBench: a tool for evaluating and synthesizing multimedia and communications systems. In Micro-30, 30th annual ACM/IEEE international symposium on Microarchitecture, pages 330-335, 1997.

[18] Jin Lin, Tong Chen, Wei-Chung Hsu, and Pen-Chung Yew. Speculative register promotion using advanced load address table (alat). In International Symposium on Code Generation and Optimization, pages 125-133, 2003.

[19] John Lu and Keith D. Cooper. Register promotion in C programs. In Proceedings of the 1997 ACM SIGPLAN conference on Programming language design and implementation, pages 308-319. ACM Press, 1997.

[20] Alexandru Nicolau. Run-time disambiguation: coping with statically unpredictable dependencies. IEEE Transactions on Computers, 38(5):633-678, 1989.

[21] Matt Postiff, David Greene, and Trevor N. Mudge. The store-load address table and speculative register promotion. In International Symposium on Microarchitecture, pages 235-244, 2000.

[22] A. V. S. Sastry and Roy D. C. Ju. A new algorithm for scalar register promotion based on SSA form. In Proceedings of the ACM SIGPLAN '98 conference on Programming Language Design and Implementation, pages 15-25. ACM Press, 1998.

[23] Standard Performance Evaluation Corp. SPEC CPU95 Benchmark Suite, 1995.

[24] Standard Performance Evaluation Corp. SPEC CPU2000 Benchmark Suite, 2000.

[25] Robert P. Wilson, Robert S. French, Christopher S. Wilson, Saman P. Amarasinghe, Jennifer M. Anderson, Steve W. K. Tjiang, Shih-Wei Liao, Chau-Wen Tseng, Mary W. Hall, Monica S. Lam, and John L. Hennessy. SUIF: An infrastructure for research on parallelizing and optimizing compilers. In ACM SIGPLAN Notices, volume 29, pages 31-37, December 1994.

[26] Robert P. Wilson and Monica S. Lam. Efficient context-sensitive pointer analysis for $\mathrm{C}$ programs. In Proceedings of the ACM SIGPLAN 1995 conference on Programming language design and implementation, pages 1-12. ACM Press, 1995. 